\title{
CONTRIBUIÇÃO DE DIFERENTES ATIVIDADES PARA O PROCESSO DE APRENDIZAGEM, NA PERCEPÇÃO DE ALUNOS DE MEDICINA
}

\author{
CONTRIBUTION OF DIFFERENT ACTIVITIES TO THE LEARNING \\ PROCESS IN THE VIEW OF MEDICAL STUDENTS \\ José Fernando de Castro Figueiredo'; Maria de Lourdes Veronese Rodrigues²; \\ Ana Raquel Lucato Cianflone ${ }^{3}$; Maria de Fátima Aveiro Colares ${ }^{4}$
}

Docentes $^{1,2}$ dos Departamentos: Clínica Médica ${ }^{1}$; Oftalmologia e Otorrinolaringologia ${ }^{2}$, Pedagoga do CAEP ${ }^{3}$ Psicóloga do CAEP4 ${ }^{4}$ da Faculdade de Medicina de Ribeirão Preto da Universidade de São Paulo.

CorRespondÊnCIA: José Fernando de Castro Figueiredo - Divisão de Moléstias Infecciosas - Departamento de Clínica Médica da Faculdade de Medicina de Ribeirão Preto - Campus Universitário - CEP: 14048-900 - Ribeirão Preto - SP

FIGUEIREDO JFC et al. Contribuição de diferentes atividades para o processo de aprendizagem, na percepção de alunos de medicina. Medicina, Ribeirão Preto, 29: 383-388, out./dez. 1996

RESUMO: Com o objetivo de conhecer o grau de contribuição de diferentes atividades para o processo de aprendizagem, na visão dos alunos da Faculdade de Medicina de Ribeirão Preto da Universidade de São Paulo, foi realizado um levantamento entre os estudantes do primeiro ao quarto anos dessa Faculdade, através da análise de item específico de um questionário padronizado, auto-administrado, e sem identificação nominal. A análise dos resultados permitiu verificar que a estratégia de ensino, "Aula Teórica", foi percebida como de média ou grande importância para o aprendizado, por aproximadamente $75 \%$ dos alunos, em todos os anos acadêmicos; no que diz respeito à estratégia "Aula Prática no Ciclo Básico" a percepção de sua importância diminui à medida em que o aluno avança no curso e a grande modificação nesse perfil ocorre na passagem do segundo para o terceiro ano. A estratégia de ensino "Aula Prática no Ciclo Clínico" foi considerada, maciçamente, como de grande importância; em relação às estratégias de aprendizagem, houve aumento na valorização do "Estudo Individual", a partir do segundo ano, e uma diminuição na importância do "Estudo em Grupo", a partir do terceiro ano. A opinião dos alunos, segundo o gênero, permitiu verificar pequenas diferenças na percepção quanto ao grau de importância de alguns itens, em diferentes anos acadêmicos.

UNITERMos: Educação Médica. Educação de Pré-Graduação em Medicina. Estudantes de Medicina - Aprendizagem.

\section{INTRODUÇÃO}

Há longo tempo, a Faculdade de Medicina de Ribeirão Preto (FMRP) se preocupa em discutir e analisar modificações do currículo do curso de graduação, com o objetivo de adaptá-lo às exigências do ensino médico moderno ${ }^{1}$. Dessa forma, iniciou a implementação de alterações em sua estrutura curricular, no ano de 1993, quando procurou, através da reorganização e da redistribuição do conteúdo, promover uma maior integração de conhecimentos². No entanto, não se verificaram modificações na maneira de administrá-los, persistindo o modelo de ensino centrado no professor e na transmissão de conteúdos.

Esse modelo de ensino caracteriza-se pelo predomínio de aulas expositivas e de práticas fragmentadas e pelo alto grau de dependência intelectual e afetiva dos alunos em relação ao professor ${ }^{3,4}$, com reflexos na forma de organização do aprendizado, por parte dos alunos. Esta forma de ensino pode estimular a pas- 
sividade e acentuar a competitividade entre os estudantes. Por sua vez, a competitividade interfere no relacionamento interpessoal, podendo levar os alunos a preferir o estudo individual em detrimento do estudo em grupo. Esse fato foi observado por Cianflone \& Fernandez ${ }^{5}$, no nosso meio, em época anterior à implantação da reforma curricular na FMRP.

Diferentes modelos de ensino têm sido empregados em outras escolas médicas, no sentido de deslocar o foco do aprendizado para o aluno, dando a ele maior responsabilidade para com o seu aprendizado ${ }^{6}$, com diferentes graus de receptividade. Esses modelos se baseiam na minimização de aulas teóricas e no estímulo ao aprendizado em pequenos grupos e por meio de interação, exigindo um corpo discente que se adapte a essa forma de aprendizagem. Assim, seria oportuno conhecer como os alunos da nova estrutura curricular da FMRP têm percebido e, dessa forma, como avaliam o grau de importância, para o seu aprendizado, de cada uma das principais atividades de ensino/aprendizagem a que estão expostos em um currículo tradicional.

\section{METODOLOGIA}

\section{Instrumento}

O levantamento da opinião dos alunos foi feito através da análise de um item específico de questionário padronizado (sem identificação nominal, auto-administrado) que tem sido aplicado pelo Centro de Apoio Educacional e Psicológico da Faculdade de Medicina de Ribeirão Preto (CAEP), com o obje- tivo de conhecer as características e necessidades da população discente dessa Faculdade. Tal item é assim constituído: "Enumere de 1 a 5, em ordem decrescente de prioridade, as atividades abaixo discriminadas, levando em conta a contribuição efetiva que cada uma delas tem para o seu aprendizado: (por exemplo: a atividade mais importante recebe 1 , a próxima 2 e assim por diante. Pode haver repetição de números)".

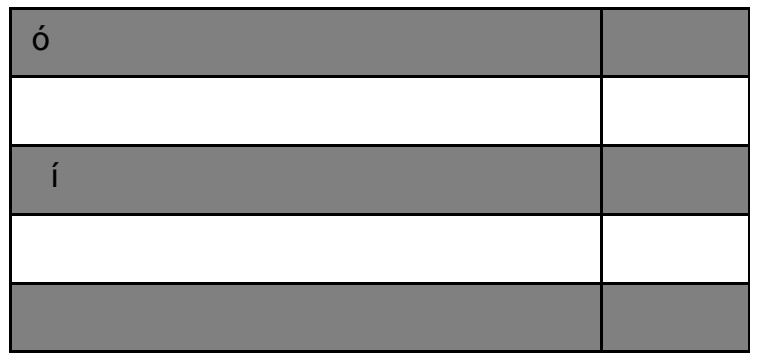

\section{Casuística}

A população de referência constituiu-se dos alunos regularmente matriculados do primeiro ao quarto anos da FMRP-USP. A população de estudo constituiu-se pelos alunos que responderam ao quesito específico do instrumento. As relações entre a população de estudo e a população de referência, segundo o ano acadêmico, estão expressas na tabela seguinte:

Uma vez que o estudo visou à otenção de dados relacionados com a nova estrutura curricular, foram excluídos os alunos do $5^{\circ}$ e $6^{\circ}$ anos, pelo fato de que estes ainda fazem parte da estrutura curricular "antiga".

Tabela I - Relações entre a população de estudo e a população de referência, segundo o ano acadêmico

\begin{tabular}{cccc}
\hline Ano Acadêmico & População de Referência & População de Estudo & $\%$ de respondentes \\
\hline $1^{\circ}$ ano & 104 & 86 & 82,7 \\
$2^{\circ}$ ano & 90 & 84 & 93,3 \\
$3^{\circ}$ ano & 104 & 78 & 75,0 \\
$4^{\circ}$ ano & 83 & 68 & 81,9 \\
\hline
\end{tabular}

\section{Análise dos Dados}

Para a análise das opções "Aulas Teóricas"; "Aulas Práticas no Ciclo Básico"; "Estudo Individual Extra-Classe" e "Estudo em Grupo Extra-Classe" foram analisadas as anotações de todos os respondentes. Levando-se em conta que as atividades práticas do Ciclo Clínico se iniciam, na FMRP, a partir do ter- ceiro ano, para a opção “Aulas Práticas no Ciclo Clínico" foram analisadas somente as respostas dos alunos do $3^{\circ}$ e $4^{\circ}$ anos. O questionário foi aplicado para todos os alunos durante o mês de setembro de 1996.

Uma vez que o instrumento previa ordem de prioridade decrescente, variando de 1 a 5 , as respostas foram agrupadas de forma a caracterizar os seguintes graus de 
relevância das atividades: 1 e 2 = grande importância; 3 = média importância; 4 e $5=$ pequena importância.

Os dados foram expressos em porcentagem simples e a comparação entre os grupos foi feita através de teste do qui-quadrado, com nível de significância de 0,05.

Para a análise das diferenças entre os gêneros, os resultados foram avaliados através das medianas das respostas para cada item, numa escala de cinco pontos, conforme metodologia utilizada por Rodrigues ${ }^{7}, 1978$.

\section{RESULTADOS}

As opiniões dos alunos sobre a contribuição de cada uma das atividades para o aprendizado, de forma global, estão representadadas nas Figuras 1 a 5.

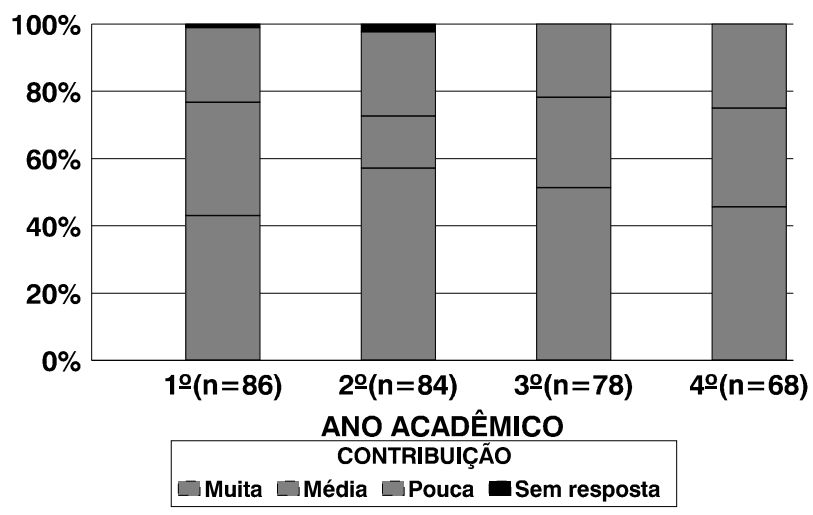

Figura 1 - Contribuição da estratégia de ensino "Aula Teórica" para o aprendizado, na percepção dos alunos da FMRP-USP.

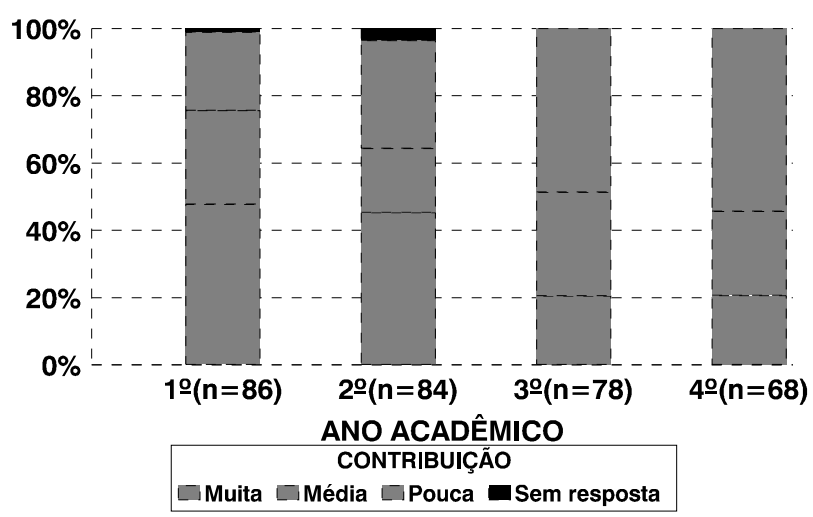

Figura 2 - Contribuição da estratégia de ensino "Aula Prática no Ciclo Básico" para o aprendizado, na percepção dos alunos da FMRP-USP.

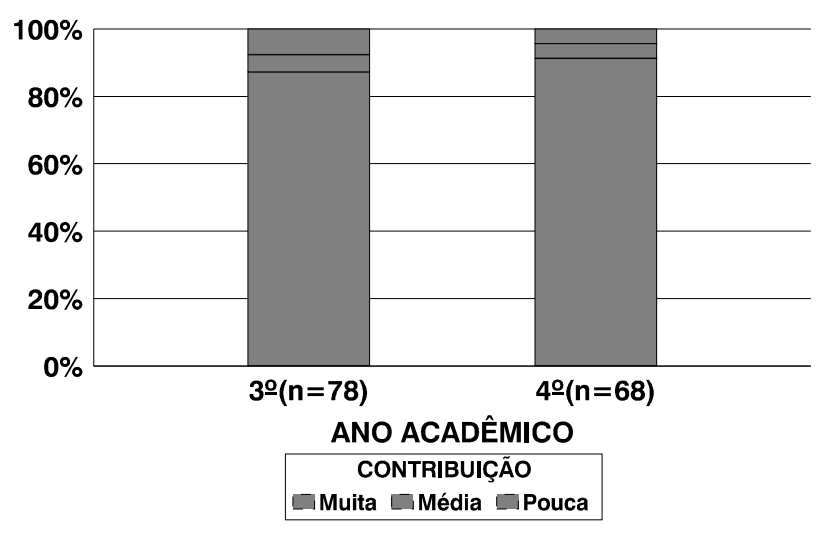

Figura 3 - Contribuição da estratégia de ensino "Aula Prática no Ciclo Clínico" para o aprendizado, na percepção dos alunos da FMRP-USP.

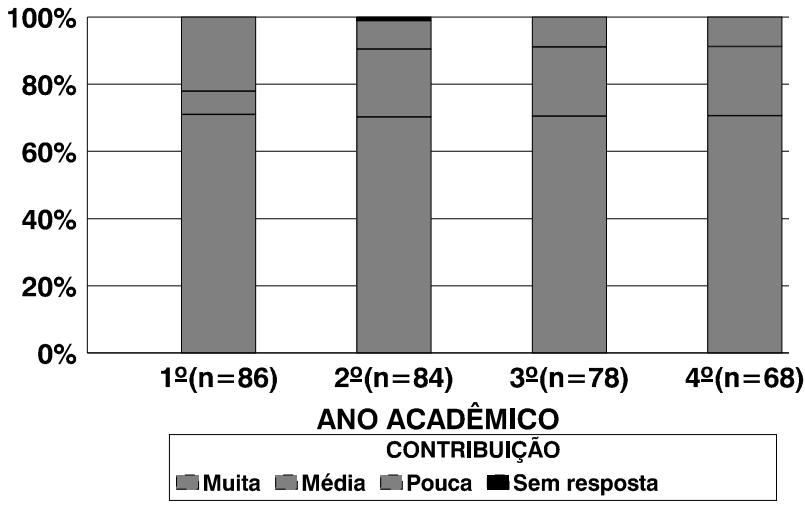

Figura 4 - Contribuição da estratégia "Estudo Individual" para o aprendizado, na percepção dos alunos da FMRP-USP.

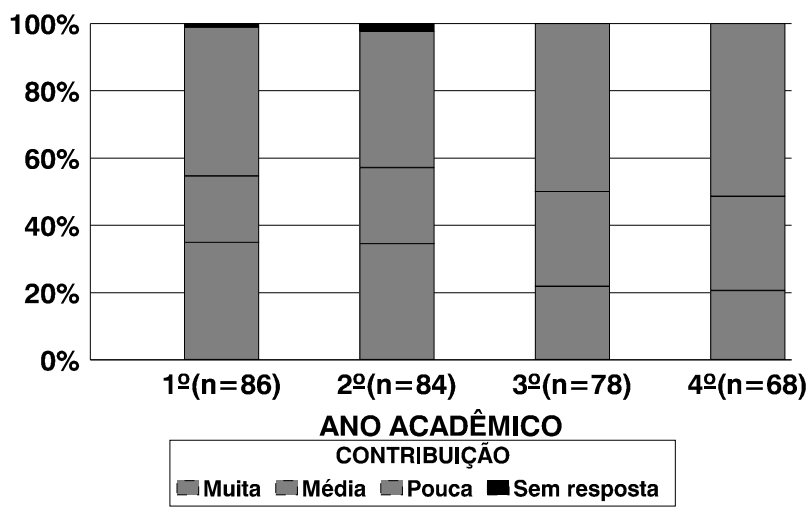

Figura 5 - Contribuição da estratégia "Estudo em Grupo" para o aprendizado, na percepção dos alunos da FMRP-USP. 
A comparação entre os gêneros, no que diz respeito à percepção da importância de cada estratégia para o aprendizado, foi avaliada através das medianas das respostas para cada ítem do questionário. As medianas foram calculadas a partir da escala original de cinco pontos, considerando cada ponto isoladamente, sem agrupamentos (Figura 6).

\section{DISCUSSÃO}

Considerando que, em estudos que utilizam questionários auto-administrados, a porcentagem de recusa é elevada ${ }^{7}$, o nível de participação dos graduandos da FMRP foi satisfatório e representativo de cada grupo de alunos (75 a 93,3\% da população de referência), e semelhante ao obtido por Cianflone \& Fernandez em $1993^{5}$.

Na Figura 1, verificamos que a estratégia de ensino "Aula Teórica "foi percebida como de média ou grande importância para o aprendizado, por aproximadamente $75 \%$ dos alunos, não havendo diferença, nessa percepção, de acordo com o ano acadêmico. Resultados semelhantes foram obtidos por Brito-Garcia et al. ${ }^{8}$ em estudo realizado na FMRP, do qual participaram estudantes de todas as séries da graduação, além de médicos residentes.

Por outro lado, no que diz respeito à importância da estratégia "Aula Teórica" para o aprendizado, no estudo de Cianflone \& Fernandez ${ }^{5}$ o item "Aula Teórica" foi considerado, quando comparado com "Estudo Individual" ou "Aulas Práticas", de menor relevância.

Com relação à estratégia de ensino "Aula Prática no Ciclo Básico" (Figura 2), verificamos que a percepção de sua importância para o aprendizado decresce à medida que o aluno avança no curso, passando de $75 \%$ de percepções como de grande ou média importância, no primeiro ano, para menos de 50\%
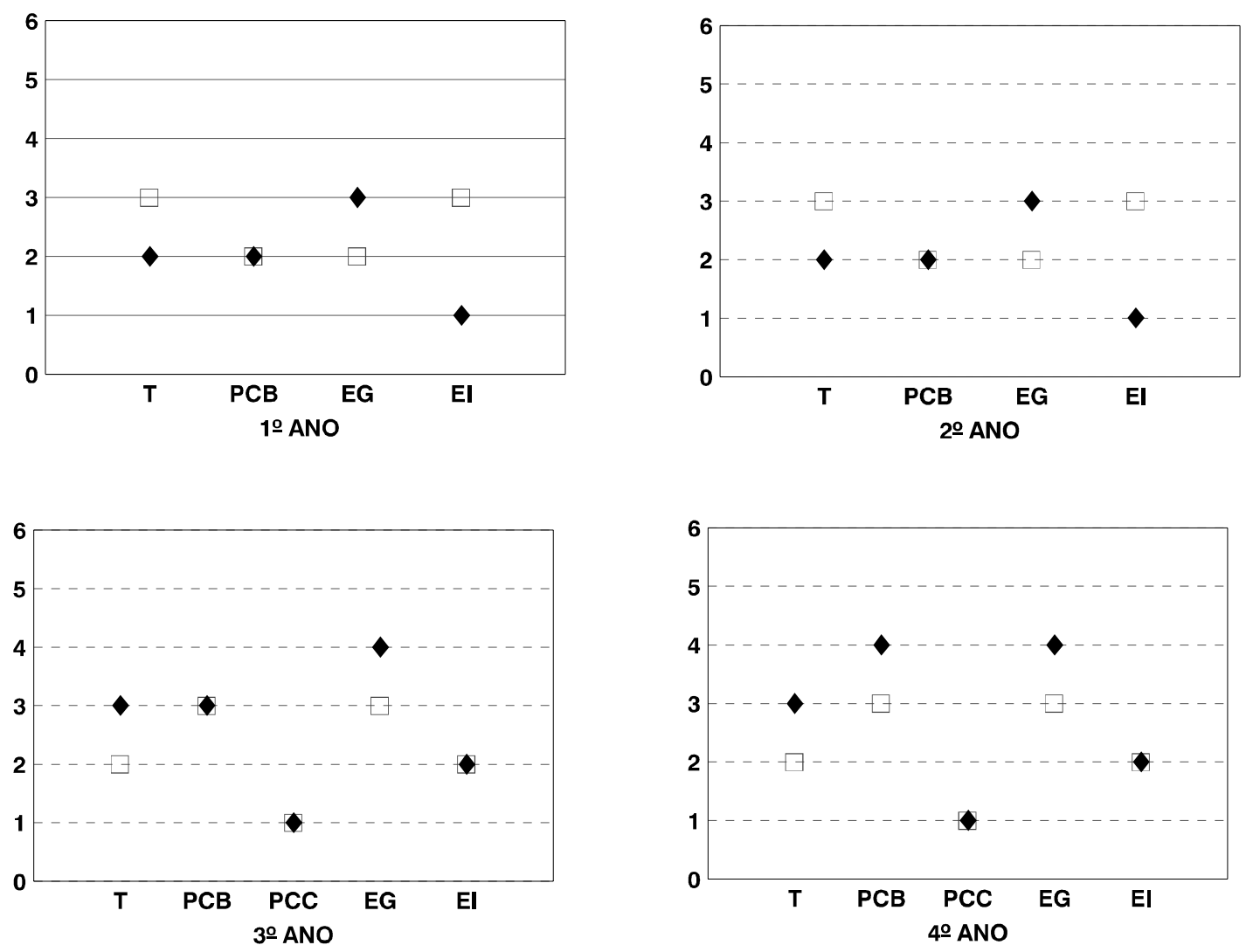

Feminino

Figura 6 - Medianas das respostas para cada ítem do questionário, de acordo com o gênero e com o ano acadêmico. 
nesses escores entre os alunos do quarto ano. Nota-se ainda que a grande modificação nesse perfil ocorre na passagem do segundo para o terceiro ano, coincidindo com o início das atividades clínicas dos alunos. Em relação à estratégia de ensino "Aula Prática no Ciclo Clínico", os alunos a consideram, maciçamente, como de grande importância, em torno de $90 \%$ desse escore (Figura 3), fato que demonstra que, na percepção dos alunos, essa metodologia é a mais relevante para a aquisição do conhecimento médico. As razões para a diminuição da importância atribuída à "Aulas Práticas no Ciclo Básico", com o passar do tempo, são obscuras. Uma delas poderia ser a dissociação entre a utilização dessa atividade com o momento em que foi formulada a questão, podendo o "esquecimento" ter relegado essa atividade a um plano secundário ${ }^{9}$.

Em relação às estratégias de aprendizagem "Estudo Individual" e "Estudo em Grupo" (Figuras 4 e 5), podemos verificar que quanto ao "Estudo individual", a partir do segundo ano houve maior valorização dessa atividade $(\mathrm{p}=0,013$; qui quadrado=6,21). Esses resultados estão de acordo com os verificados por Brito-Garcia et al. ${ }^{8}$, cujos respondentes elegeram o estudo individal como o melhor método de aprendizagem. Por outro lado, em relação ao "Estudo em Grupo", a partir do terceiro ano, houve uma tendência de diminuição do percentual de alunos que o consideravam de grande importância ( $\mathrm{p}=0,07$; qui-quadrado $=3,22$ ). A tendência para a individualização do estudo poderia ser reveladora de alterações nas relações sociais intraclasses $^{4}$, em especial a competitividade ${ }^{5,9}$.
A análise das opiniões dos alunos segundo o gênero (Figura 6), permite verificar pequenas diferenças de percepção quanto ao grau de importância dos diferentes itens estudados, entre homens e mulheres, em diferentes anos acadêmicos. Assim, por exemplo, no primeiro ano, a atividade "Estudo Individual" foi mais valorizada pelos homens; no segundo ano a atividade "Aulas Práticas no Ciclo Básico" foram consideradas, também, de maior relevância pelos homens. Em relação aos terceiro e quarto anos, a distribuição das respostas foi bastante similar entre os dois gêneros e para todas as atividades avaliadas.

\section{CONCLUSÕES}

Do exposto acima, pode-se depreender que os alunos da FMRP valorizam positivamente as atividades de ensino "Aula Teórica" e "Aula Prática no Ciclo Clínico", ao passo que a atividade de ensino "Aula Prática no Ciclo Básico" se situa em patamar inferior de relevância. Podemos concluir também que o perfil predominante do aluno aponta para a preferência do estudo individual sobre o estudo em grupo.

Cabe ressaltar que os resultados obtidos poderiam ser o reflexo da não exposição dos alunos a outras metodologias de ensino/aprendizagem, nas quais os alunos têm uma participação mais ativa, com maior grau de interação e cooperação. Nessa perspectiva, o estudo em grupo tem forma e enfoques diferentes e os próprios grupos são formados levando em conta, também, critérios pedagógicos.

FIGUEIREDO JFC et al. Contribution of different activities the learning process in the view of medical students. Medicina, Ribeirão Preto, 29: 383-388, oct./dec. 1996

ABSTRACT: To determine the extent of the contribution of different activities to the learning process in the view of students at the Faculty of Medicine of Ribeirão Preto, University of São Paulo, a survey was conducted on first to fourth year medical students by the analysis of a specific item in a standardized self-administered questionnaire with no identification of respondent names. Analysis of the results showed that the "Lecture" teaching method was perceived to be of medium or high importance by approximately $75 \%$ of the students in all years of the medical course. The perception of the importance of "Laboratory Class of the Basic Cycle", decreased with the year of medical course, with the most significant modification of this profile occurring in the passage from the second to the third year. The teaching method "Clinical Activities" was massively considered to be of great importance for learning. With respect to learning methods, there was an increase in the appreciation of "Individual Study" from the second year on and a decrease in the importance of "Group Study" from the third year on. Small differences between gender were observed in terms of the perception of the importance of some items studied in different years of medical course.

UNITERMS: Education, Medical. Education, Medical, Undergraduate. Students, Medical. Learning. 


\section{REFERÊNCIAS BIBLIOGRÁFICAS:}

1 - FIGUEIREDO JFC \& RODRIGUES CRC. Estratégias para a reformulação da estrutura curricular da Faculdade de Medicina de Ribeirão Preto na última década e estágio atual do processo. Rev Bras Educ Med 14: 20-23, 1990.

2 - FACULDADE DE MEDICINA DE RIBEIRÃO PRETO DA UNIVERSIDADE DE SÃO PAULO. Processo 94.1.1288.17.8, v.3. Estrutura Curricular.FMRP, 1993/1996.

3 - BARROWS HS \& TAMBLYM RM. Problem- based learning: an approach to medical education. Springer Publishing, New York, 1980. 206 p.

4 - MIZUKAMI MGN. Ensino: as abordagens do processo. EPU, São Paulo, 1986. 119p.

5 - CIANFLONE ARL \& FERNANDEZ JM. Algumas características do ensino e aprendizado na Faculdade de Medicina de Ribeirão Preto - USP - um estudo junto aos alunos de graduação. Medicina, Ribeirão Preto, 26: 228-236, 1993.
6 - FIGUEIREDO JFC. Relatório. Universidade de São Paulo/Faculdade de Medicina de Ribeirão Preto, 1995 (visita à Harvard Medical School).

7 - RODRIGUES CRC. Contribuição ao estudo dos objetivos educacionais em Psicologia Médica e Psiquiatria. Dissertação de Mestrado, Faculdade de Medicina de Ribeirão Preto da USP, p. 1-111, 1978.

8 - BRITO-GARCIA S et al. Opinião dos alunos da FMRP-USP sobre a eficácia de diferentes métodos de ensino utilizados no curso médico. In: Anais, XXX Congresso Brasileiro de Educação Médica. Associação Brasileira de Escolas Médicas, Porto Alegre, p 57, 1995.

9 -MILLER GE. org. Ensino e aprendizagem nas escolas médicas. São Paulo, Editora Nacional, 1967. 330 p.

Recebido para publicação em 23/10/96

Aprovado para publicação em 11/12/96 